\title{
Numerical Applications of Reflection to Partial Differential Equations
}

\author{
By Arthur David Snider*
}

\begin{abstract}
Recent papers have reported results on the numerical solution of nonlinear free boundary problems wherein a conformal transformation (which must be determined) maps the unknown flow region on to a known domain; the boundary conditions are handled by the method of steepest descent. The present paper discusses the use of the reflection property of solutions of elliptic equations to determine these boundary conditions. The procedure is applied to the vena contracta models, and it is seen that it converges about ten times faster than the steepest-descent method.
\end{abstract}

This paper will report the results of a study of the application of the procedure of analytic continuation to the numerical solution of partial differential equations, and in particular, to the vena contracta problem.

The possibility of extending the solution of an analytic boundary value problem across the boundary has been explored in [1], [2], and [3]. The results show that in many cases (including nonlinear problems), there is a formula expressing the solution at points beyond the boundary in terms of the values in the original domain; such an expression is commonly called a reflection rule. Here we shall examine in detail the theory behind the reflection scheme for the problem of finding a conformal transformation with certain boundary restrictions.

This concept has an immediate application in the numerical solution of free boundary problems, such as the vena contracta [4], [5]. The conformal map is used to transform the complicated, unknown domain of the solution to a simpler, known domain, wherein the (transformed) differential equation can be more easily solved; of course, one must solve for the transformation simultaneously. The result is then regarded as a parametrized form of the solution. The principal difficulty in this procedure is the handling of the boundary conditions for the conformal map. In [4] Bloch derived boundary equations from a steepest-descent argument, supplemented by equations serving to establish the free boundary constraint (which in the case of the vena contracta expresses continuity of the pressure across a fluid-air interface). Our paper purports to demonstrate that the reflection technique is superior to the method of steepest descent in handling these problems.

Part 1 of this paper describes the theory behind this reflection scheme. Proofs of certain results are given when available, and numerical evidence of other consequences

\footnotetext{
Received May 18, 1972; revised June 18, 1975.

AMS (MOS) subject classifications (1970). Primary 30A28, 35-04; Secondary 65M05, 76B10.

Key words and phrases. Reflection, free boundary, finite differences, conformal mapping.

* The author wishes to acknowledge the advice and guidance of his thesis advisor, Paul R. Garabedian, in the preparation of this paper. 
is offered so that a heuristic understanding of the scheme's implications and limitations is obtained.

The application of the procedure in solving the vena contracta comprises Part 2.

Some theoretical aspects peculiar to free-surface problems are studied; furthermore, this model is full of complications not related to reflection and each of these must be dealt with. In particular, we present some special techniques for handling the point of separation of the free surface from the wall.

Part 3 describes the results of programming this procedure on New York University's CDC 6600 computer. The accuracy and efficiency are reported, and it is shown that the reflection method converges about ten times faster than the steepest-descent procedure mentioned above. New calculations of the two- and three-dimensional contraction coefficients are quoted; they agree extremely well with those of Bloch [4].

The evidence offered herein clearly demonstrates the superiority of this scheme in handling the vena contracta model, and we are led to conclude that reflection is the appropriate method for solving a wider class of free boundary problems which arise in fluid dynamics and plasma physics. Furthermore, the results tend to encourage the use of reflection rules whenever they are applicable in solving more general nonlinear boundary value problems in two independent variables.

Part 1. Reflection. In [1] Lewy shows that the solutions to many two-dimensional analytic elliptic differential equations, subject to analytic boundary conditions, can be continued across the boundary in a prescribed manner. We shall demonstrate how this concept may be used to develop a finite-difference method for finding conformal maps.

A. Calculation of Conformal Maps. Let $z(w)=x(u, v)+i y(u, v)$ be an analytic function mapping, say, a rectangle in the $w$-plane onto a domain $D$ in the $z$-plane. The equations below follow from the Cauchy-Riemann conditions:

$$
\begin{gathered}
\Delta x=0, \\
\Delta y=0, \\
x_{u} x_{v}+y_{u} y_{v}=0, \\
1 / 2\left(x_{v}^{2}+y_{v}^{2}-x_{u}^{2}-y_{u}^{2}\right)=0 .
\end{gathered}
$$

Direct computation will verify the following observations. If two functions $x$ and $y$ satisfy (1) and (2), then the left-hand side of (3) will be a harmonic function, whose harmonic conjugate is the left-hand side of (4) (hence, the factor $1 / 2$ ). Thus, if (3) is satisfied on the boundary of the rectangle, it will hold true everywhere; and consequently, the function in (4) will be constant. Furthermore, the equations (1) through (4) imply that $z(w)$ is conformal (analytic or anti-analytic; the latter possibility is easily eliminated in numerical schemes by initialization, and we consequently ignore it).

This suggests a procedure which will yield conformal transformations: find harmonic functions $x$ and $y$ of the variables $u$ and $v$ such that (3) holds on the boundary of the region and (4) holds at one, arbitrary, point (see also [4]). The scheme presented herein is based on this strategy.

B. Application of the Reflection Rules. To implement the above numerically, we 
set up a mesh of uniform size $h$ on, say, a rectangle in the $w$-plane, which is to be mapped onto a region in the $z$-plane bounded by analytic curves. Then we can write the finite-difference analogue of Laplace's equation at the interior mesh points for the functions $x(u, v)$ and $y(u, v)$ :

$$
x_{i, j}=1 / 4\left(x_{i+1, j}+x_{i-1, j}+x_{i, j+1}+x_{i, j-1}\right)
$$

(to order $O\left(h^{2}\right)$ ). Now we must write equations for $x$ and $y$ on the boundary.

According to the theory (cf. [1], [3], [6]), these harmonic functions can be analytically continued across the boundary for some distance. Our technique, then, is to define $x$ and $y$ at new mesh points just outside the rectangle and use these to write Eq. (5) along the boundary, as well as in the interior (see Fig. 1). Now the problem becomes one of finding reflection rules, based on the boundary conditions, which will determine the values of $x$ and $y$ at the exterior mesh points.
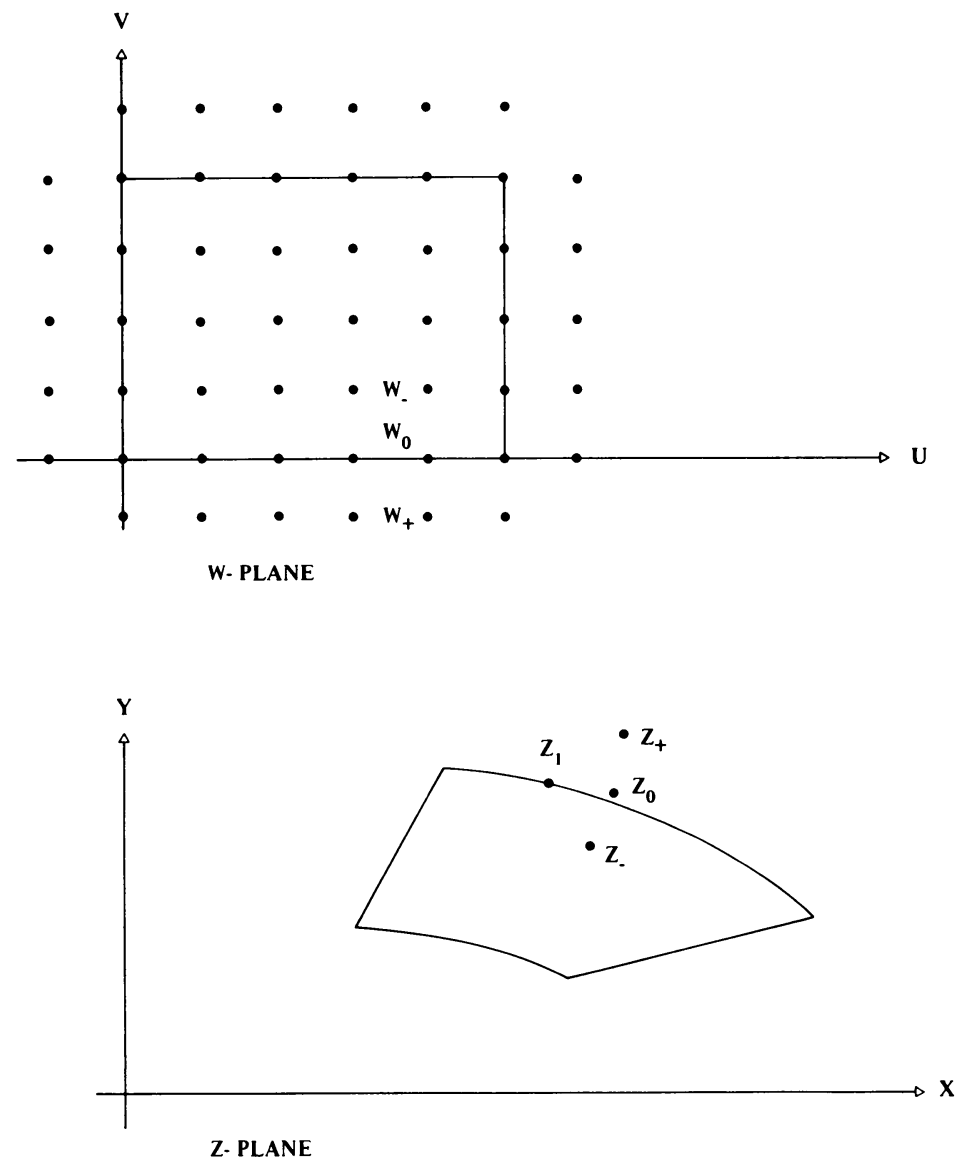

FiguRE 1

The boundary conditions demand that the sides of the rectangle map onto the analytic curves bounding the image region in the $z$-plane. For definiteness, let us say that the pre-image of the analytic curve $\Gamma$ is the bottom of the rectangle, $v=0$ (again see Fig. 1). 
The equation for $\Gamma$ has the form

$$
g(x, y)=0,
$$

where $g$ admits a power series expansion. Making the substitution

$$
x=(z+\bar{z}) / 2, \quad y=(z-\bar{z}) / 2 i,
$$

we get the equation for $\Gamma$ in the form

$$
F(z, \bar{z})=0
$$

with $F\left(z_{1}, z_{2}\right)$ complex-analytic in $z_{1}$ and $z_{2}$. The reflection rule now is given by the following theorem.

THEOREM. Let $z(w)$ be an analytic function defined on both sides of the line $v=0$, mapping this line onto the analytic curve given by (8). If $w_{-}$is an interior point of the rectangle and $w_{+}$is its mirror image reflected through the line $v=0$, and if $z_{-}=z\left(w_{-}\right)$and $z_{+}=z\left(w_{+}\right)$, then

$$
F\left(z_{+}, \bar{z}_{-}\right)=0 \text {. }
$$

Equation (9) tells how to compute $x$ and $y$ at the exterior mesh points. Notice its validity when $\Gamma$ is one of the axes, or a circle.

Proof of (9). Observe that the function $f(w)=F(z(w), \overline{z(\bar{w}))}$ is analytic. It vanishes on the line $v=0$, hence is identically zero. Setting $w=w_{+}$, Eq. (9) results.

Clearly, the same Eq. (9) holds for reflection through the other sides of the rectangle, so it provides the general rule for analytic continuation, and we now have a feasible scheme for computing conformal maps: we write (5) for $x$ and $y$ at the interior and boundary mesh points, and we write (9) at the exterior points. The next section discusses the consequences of using this procedure.

C. Implications of the Reflection Rules. For the moment let us assume that the solution to this system of equations exists and is unique for every sufficiently small mesh size $h$, and that the solution converges to a function $z(u, v)=x+i y$, where $x$ and $y$ are harmonic, as $h$ goes to zero. These assumptions will be discussed in the next section. What are the properties of $z$ on the boundary?

Let us examine a particular point $w_{0}$ on the boundary of the rectangle, together with its inner neighbor $w_{-}$and its reflected neighbor $w_{+}$(see Fig. 1). As $h$ goes to zero, the three points $z_{+}=z\left(w_{+}\right), z_{0}=z\left(w_{0}\right), z_{-}=z\left(w_{-}\right)$, must all converge to the same point $z_{1}$. But $F\left(z_{+}, \bar{z}_{-}\right)=0$, so $F\left(z_{1}, \bar{z}_{1}\right)=0$ and $z_{1}$ lies on $\Gamma$. (Another way of seeing this is to notice that $z_{+}$and $z_{-}$always lie on opposite sides of $\Gamma$, so $z_{1}$ must lie on $\Gamma$.) We conclude that the limiting function $z$ maps the boundary of the rectangle onto the boundary of the region in the $z$-plane.

Now it is our goal to prove that the normal and tangential derivatives on the sides of the rectangle of $z$ are perpendicular, i.e., $x_{n} x_{t}+y_{n} y_{t}=0$. To this end we must study the geometry of the curve $\Gamma$.

First, we assume that the function $F\left(z_{1}, z_{2}\right)$ was derived from a real function $g(x, y)$ as described earlier, so that $F(z, \bar{z})$ is always real. If $z$ is given as a function of a real variable $s, z=z(s)$, then $d F / d s$ must be real. Since 


$$
d F / d s=F_{1} z_{s}+F_{2} \bar{z}_{s},
$$

we conclude

$$
F_{2}(\zeta, \bar{\zeta})=\overline{F_{1}(\zeta, \bar{\zeta})}
$$

Now, if $z(t)$ is a parametrization of the curve $\Gamma, F(z(t), \overline{z(t)})=0$. Thus,

$$
\begin{aligned}
d F / d t=0 & =F_{1} z_{t}+F_{2} \bar{z}_{t}=\bar{F}_{2} z_{t}+F_{2} \bar{z}_{t}=2 \operatorname{Re}\left(F_{2} \bar{z}_{t}\right), \\
0 & =\left(\operatorname{Re} F_{2}\right) x_{t}+\left(\operatorname{Im} F_{2}\right) y_{t} .
\end{aligned}
$$

If we associate, with each complex number, a vector whose $x$ and $y$ components are the real and imaginary parts, respectively,(12) says that $F_{2}(\zeta, \bar{\zeta})$ is normal to the curve $F(\zeta, \bar{\zeta})=0$ (since the tangent is $\left(x_{t}, y_{t}\right)$ ).

We can get more information about $\Gamma$ by applying the analytic form of the implicit function theorem. If $F\left(z_{1}, z_{2}\right)$ is analytic in both variables with $F(a, b)=0$ and $F_{1}(a, b) \neq 0$, then $F\left(z_{1}, z_{2}\right)=0$ defines $z_{1}$ as an analytic function of $z_{2}$ in a neighborhood of $(a, b)$;

$$
\left(z_{1}-a\right)=c_{0}+c_{1}\left(z_{2}-b\right)+c_{2}\left(z_{2}-b\right)^{2}+\cdots .
$$

Furthermore, $c_{0}=0$ and $c_{1}=-\left(F_{2} / F_{1}\right)(a, b)$.

We have (9) defining $z_{+}$in terms of $z_{-}$. Let us suppose that $z_{+}$and $z_{-}$both approach $\zeta$ as $h \rightarrow 0$; we just proved $\zeta$ was on the curve $\Gamma$; so $F(\zeta, \bar{\zeta})=0$.

Equation (13) then becomes

$$
\left(z_{+}-\zeta\right)=-\frac{F_{2}(\zeta, \bar{\zeta})}{F_{1}(\zeta, \bar{\zeta})}\left(\bar{z}_{-}-\bar{\zeta}\right)+c_{2}\left(\bar{z}_{-}-\bar{\zeta}\right)^{2}+\cdots
$$

Using (11) and adding $\zeta-z_{-}$to both sides, we ultimately have

$$
z_{+}-z_{-}=-\frac{F_{2}}{\left|F_{2}\right|^{2}}\left[F_{2}\left(\bar{z}_{-}-\bar{\zeta}\right)+\bar{F}_{2}\left(z_{-}-\zeta\right)\right]+O\left(\left|z_{-}-\zeta\right|^{2}\right) .
$$

As the mesh size $h$ of the rectangle becomes smaller, the quantities $\left(\zeta-z_{-}\right) / h$ and $\left(z_{+}-z_{-}\right) / 2 h$ should give approximations to the derivative of $z$ in the direction normal to the side of the rectangle, denoted $z_{n}$. Thus, if we divide the above equations by $2 h$ and take the limit, we get

$$
z_{n}=\left(F_{2} / 2\left|F_{2}\right|^{2}\right)\left[F_{2} \bar{z}_{n}+\bar{F}_{2} z_{n}\right] \text {. }
$$

Since the coefficient of $F_{2}$ is real, the direction of the vector $z_{n}$, if it is nonzero, is the same as that of $F_{2}$, i.e. normal to the curve $\Gamma$.

Of course, the derivative $z_{t}$ of $z$ along the side of the rectangle will be parallel to the tangent to $\Gamma$; so we will have

$$
x_{n} x_{t}+y_{n} y_{t}=x_{u} x_{v}+y_{u} y_{v}=0
$$

for the function $z(u, v)$ along the boundary of the rectangle. Reasoning as before for 
these harmonic functions $x$ and $y$, we conclude that (17) holds throughout the rectangle and that $x_{v}^{2}+y_{v}^{2}-x_{u}^{2}-y_{u}^{2}=H$ is constant there.

From what we have said so far, we cannot conclude that $z$ is analytic, i.e., that $H=0$. For example, the mapping $x=2 u, y=v$ satisfies all the above requirements and is not analytic. To get an analytic map we must impose some additional condition to drive $H$ to zero; presumably the particular application will suggest such a condition (see below).

D. Existence, Uniqueness, and Convergence. We discuss the existence and uniqueness of the solution, and its convergence as $h \longrightarrow 0$, on the basis of numerical experiments.

The method has been programmed for several different cases of image regions bounded by four analytic curves. The vena contracta described below leads to just such a region. The reflection rules were applied so as to map each side of the rectangle onto one of the bounding curves. This amounts to a four-point normalization because each corner must be carried into the intersections of these curves. The equations were iterated using overrelaxation to accelerate convergence and in each case the iterates did converge. This indicates (but does not prove) that the solution of the four-point normalization problem exists and is unique (if there were two solutions, one would expect the iterates to oscillate between them). By shifting one of the curves we can get different values for $H$; only one position makes $H=0$, and at that point the finite-difference analogues of the Cauchy-Riemann equations are satisfied.

No rigorous proof of convergence of the method as $h \rightarrow 0$ has been developed, but the problem has similarities to the Dirichlet and Neumann problems, whose finitedifference analogues have been analyzed [3], [7], [8]. In the latter cases, the solutions of the difference equations approach those of the differential equation with an error of the order $O\left(h^{2}\right)$, so we expect the same of our scheme also. Numerical experiments confirm that the convergence is $O\left(h^{2}\right)$ (see below).

It can be shown that a three-point-normalized scheme actually forces $H$ to be zero; see [9] for details.

E. Summary. The results of the preceding section can be stated concisely as follows.

Consider analytic boundary curves described by equations of the form (8). We write Eq. (5) for $x$ and $y$ in a region in the $w$-plane; the equations are written for each interior and boundary mesh point. Then we write (9) for the reflected points. This procedure constitutes the reflection scheme for conformal mapping problems.

When the $w$-region is a rectangle and the image is bounded by four analytic curves, each corresponding to a side of the rectangle, our numerical experiments lead us to conjecture the following theorem.

THEOREM. The solutions $x_{h}$ and $y_{h}$ of the equations of the reflection scheme converge to harmonic functions $x(u, v)$ and $y(u, v)$ as the mesh size $h$ is diminished. The rate of convergence is $O\left(h^{2}\right)$.

Furthermore, whenever the solutions and their difference quotients converge, we have proved the following

THEOREM. Under the convergence assumptions stated, the function $z(w)$ maps 
the sides of the rectangle onto the boundary curves, and on these sides the derivatives of $z$ in the normal and tangential directions are perpendicular to each other.

Equation (3) then holds throughout the region, and the quantity $H$ is constant. Experimentation shows that the value of $H$ depends on the locations of the curves in the $z$-plane; $H=0$ can be achieved by shifting them. This condition will then ensure that $z(w)$ is analytic.

The above procedure seems to be most suitable for applications of the method of reflection when the curves in the $z$-plane are analytic and known. However, as we proceed in Part 2 it will become clear that neither of these conditions is essential, and one can often adapt the technique to less restrictive cases.

Part 2. The Vena Contracta. We now describe the application of the reflection technique in solving the two- and three-dimensional vena contracta problems.

A. The Physical Problem and Parameters. The vena contracta model is depicted in Fig. 2. An incompressible, inviscid fluid under pressure is allowed to escape as a jet stream through an aperture in the infinite containing wall. The width of the aperture is $2 Y_{0}$; the asymptotic width of the stream is $2 Y_{\infty}$. For the two-dimensional case the aperture is a slit; in three dimensions, it is a circular hole (so the problem is axially symmetric). Symmetry allows us to restrict ourselves to the upper half $(x, y)$-plane.

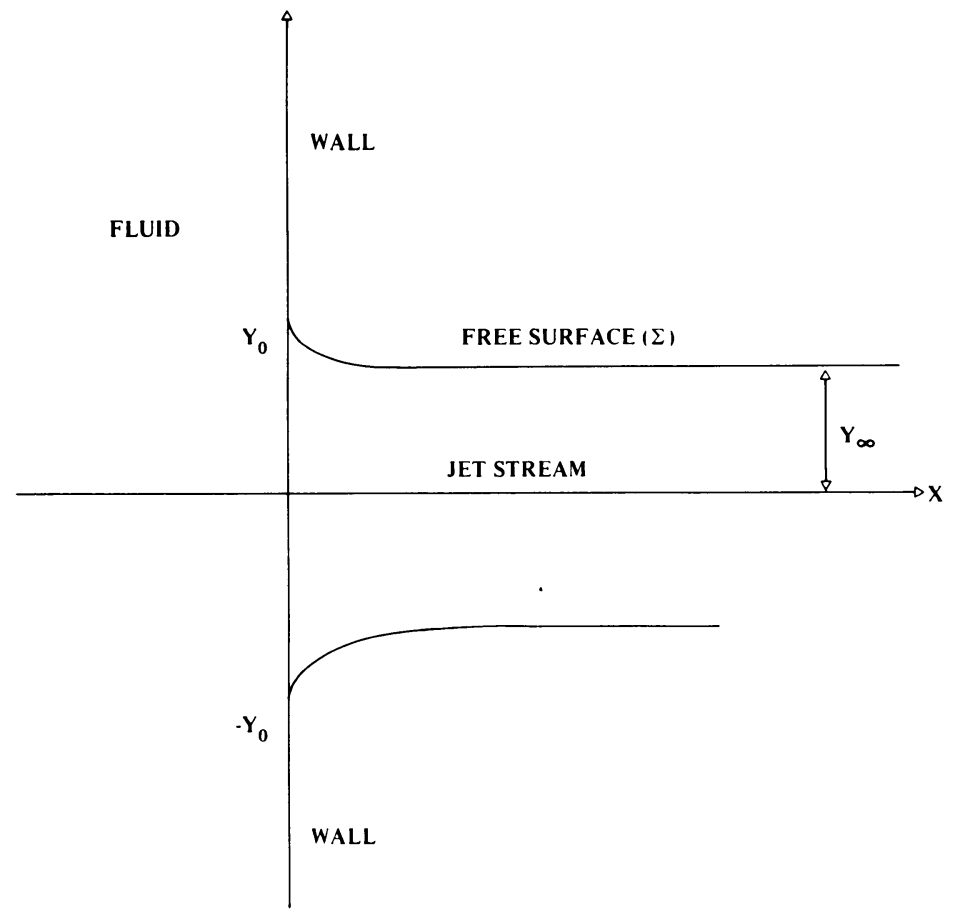

Figure 2

For details of the physics of the flow, we refer the reader to [4], [5], [9], or [10]. Here, in the interest of brevity, we merely summarize the mathematical description. Following [10], we treat the two- and three-dimensional cases simultaneously by introducing the parameter $m$ : 


$$
m= \begin{cases}0 & \text { in two dimensions, } \\ 1 & \text { in three dimensions. }\end{cases}
$$

The motion is described by the stream function $\psi(x, y)$, which gives the $x$ and $y$ components of velocity by

$$
v_{x}=(\partial \psi / \partial y) / y^{m}, \quad v_{y}=-(\partial \psi / \partial x) / y^{m} .
$$

The equation for $\psi$ is

$$
\Delta \psi-(m / y)(\partial \psi / \partial y)=0
$$

A consistent set of boundary data is

$$
\begin{gathered}
\psi=0 \text { on the } x \text {-axis. } \\
\psi=1 /(1+m) \text { on the wall and the free surface } \Sigma .
\end{gathered}
$$$$
(\partial \psi / \partial n) / y^{m}=1 \text { on } \Sigma,
$$

where we have scaled so that $Y_{\infty}=1$ (cf. [9] for details); Eq. (23) is the free surface condition on the normal derivative. In addition, we observe

$$
\psi \approx y^{1+m} /(1+m) \text { as } x \rightarrow \infty .
$$

With this normalization, the diameter $Y_{0}$ cannot be specified arbitrarily; in fact, one of our goals will be to calculate the contraction coefficient

$$
C_{c}(m)=\left(Y_{\infty} / Y_{0}\right)^{m+1} .
$$

B. The Conformal Map. To facilitate the solution of this problem we map an infinite rectangle in the $w$-plane conformally onto the (upper-half) flow region. Corresponding points for this mapping are indicated in Fig. 3; the image of $B$ is $B^{\prime}$, etc. A few comments are in order here.

Practically speaking, we must truncate the rectangle at a finite length $U_{\max }$, indicated by the line $C D$ (and $C^{\prime} D^{\prime}$, its image). $B C$ maps onto the free surface $\Sigma$; of course, the location of $\Sigma$ is unknown, and this will result in a complicated set of boundary conditions on $B C$. The corner at $A$ goes to the point at infinity in the second quadrant, so near $A$ the map has the form

$$
z(w) \approx R / w
$$

with a negative residue $R$ which must be calculated.

Since the map $z(w)$ is analytic, we can transform Eqs. (20)-(23) for $\psi$ in the $w$-plane. Equation (20) becomes

$$
\Delta \psi-m(\nabla y \cdot \nabla \psi) / y=0 .
$$

(21) holds on $A D$, (22) on $A B$ and $B C$, and the free boundary condition (23) becomes

$$
(\partial \psi / \partial v)^{2} /|\partial z / \partial v|^{2} y^{2 m}=1
$$

on $B C$ 
Now we have to find the solution $\psi(u, v)$ and the conformal map $z(w)$ simultaneously. To to this numerically, we impose a uniformly-spaced mesh (of spacing $h$ ) on the rectangle, including the set of reflection points on the sides $A B, B C$, and $A D$ (Fig. 3). We now turn to the problem of writing equations for $\psi(u, v)$ and $z(w)$ at these points.
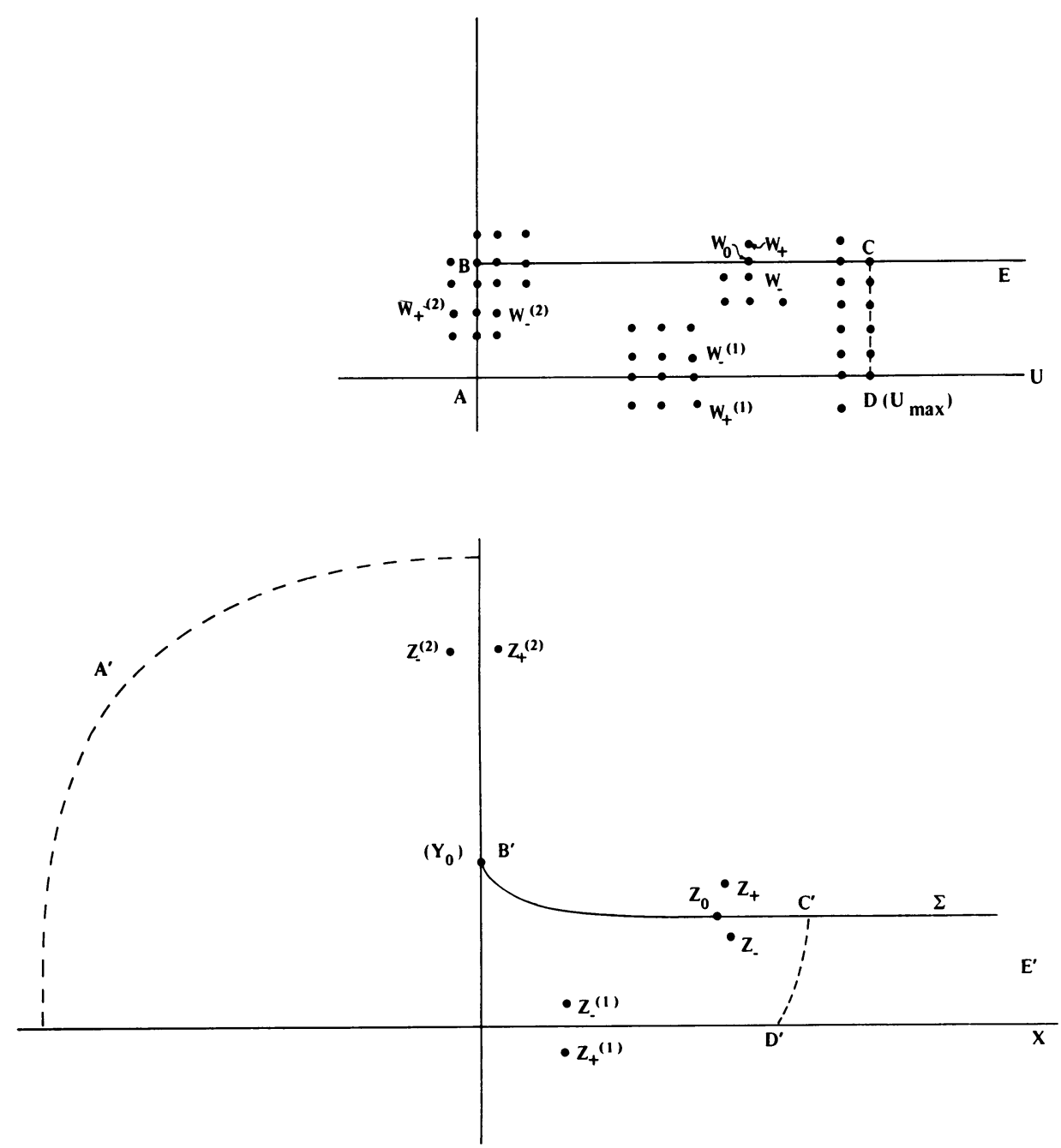

Figure 3

C. The Interior Points. Since $x$ and $y$ are harmonic functions, we write Eq. (5) for each of these at every interior mesh point. The same equation is written for $\psi$ in two dimensions. For the axially symmetric case, we approximate equation (27) by

$$
\psi_{i j}=\frac{a_{i-1, j} \psi_{i-1, j}+a_{i, j-1} \psi_{i, j-1}+a_{i+1, j} \psi_{i+1, j}+a_{i, j+1} \psi_{i, j+1}}{a_{i-1, j}+a_{i, j-1}+a_{i+1, j}+a_{i, j+1}},
$$


where

$$
a_{k, l}=1 /\left(y_{k, l}+y_{i, j}\right)
$$

which is correct to order $O\left(h^{2}\right)$ (cf. [4]). (However, see the next section for some modification near the corner $A$.)

D. The Wall and the $x$-Axis. The values of $\psi$ are specified on the wall and the $x$-axis (or $A B$ and $A D$ ) by Eqs. (21) and (22).

Equation (5) can be written for $x$ and $y$ at each mesh point on $A B$ and $A D$ if we use reflection rules to compute values at the exterior points; these are easily found by the method of Part 1 and we have

$$
y_{+}=y_{-} \quad \text { and } \quad x_{+}=-x_{-} \text {on } A B
$$

and

$$
x_{+}=x_{-} \text {and } y_{+}=-y_{-} \text {on } A D .
$$

However, we must incorporate some modifications to account for the singular natures of $\psi$ and $z$ at the corner $w=0 ; \psi$ has a jump discontinuity at $A$, while $z$ has a pole (26). We proceed exactly as in [4] for $\psi$ by subtracting off a known solution $\psi_{1}$, which incorporates the discontinuity, in a neighborhood of the origin:

$$
\psi_{1}=\frac{2}{\Pi} \arccos \left(\frac{u}{\left(u^{2}+v^{2}\right)^{1 / 2}}\right)
$$

in two dimensions, and

$$
\psi_{1}=\frac{1}{2}\left(1-\frac{u}{\left(u^{2}+v^{2}\right)^{1 / 2}}\right)
$$

in three dimensions. Furthermore, in the axially symmetric case the truncation error in Eq. (29) becomes unbounded, so we also perform a Kelvin transformation

$$
z^{\prime}=1 / z, \quad \psi^{\prime}=\psi /|z|,
$$

in the same neighborhood of $A$. See [4] for details.

The singularity in $z(w)$ is handled by subtracting off the pole and writing Eq. (5) for the (regular) function

$$
\zeta(w)=z(w)-R / w
$$

Again, this transformation is performed in a neighborhood of $A$. The reflection rules for $\zeta$ are derived from Eqs. (30) and (31); in fact, they are identical with the rules for $z$. The residue $R$ must be computed; we defer discussion of this point until section $H$.

The interfacing of this special region near $A$ with the rest of the rectangle is a simple programming matter and we again refer to [4] for the details.

E. The Reflection Law at the Free Surface. $B C$ is to be mapped onto the free boundary $\Sigma$, but the reflection-rule situation is more complicated than that treated in 
Part 1. The location of $\Sigma$ is unknown and hence we have no simple equation like (8) to work with. However, we can derive a reflection rule from the integral equation for $\psi$ in two dimensions, and we will modify it to make it suitable in three dimensions.

The implications of this new procedure will then have to be reviewed.

First, we rewrite Eq. (8) in the form

$$
\bar{z}=g(z) .
$$

Garabedian [10] has derived an integral equation relating $\psi$ and $g$. In two dimensions, it takes the form

$$
\psi(x, y)=\operatorname{Im} \int_{z_{1}}^{z} g^{\prime}(t)^{1 / 2} d t .
$$

Of course, $\psi$ is harmonic in two dimensions and thus can be combined with its harmonic conjugate $\phi(x, y)$ to give an analytic function $\zeta(z)$. Equation (37) displays this property, and we see that

$$
\zeta(z)=\phi+i \psi=\int_{z_{1}}^{z} g^{\prime}(t)^{1 / 2} d t .
$$

By differentiating, we can solve for $g(z)$ to get

$$
g(z)=\int_{z_{2}}^{z}\left(\zeta^{\prime}(t)\right)^{2} d t
$$

Now, if $z_{0}$ lies on $\Sigma$, we can write (36) for it; and if $z_{+}$is the reflected image of $z_{-}$as in Fig. 3, we can write the reflection rule derived from (36), namely

$$
\bar{z}_{+}=g\left(z_{-}\right) .
$$

Combining these, we eliminate the fiducial point $z_{2}$ to obtain

$$
\bar{z}_{+}-\bar{z}_{0}=\int_{z_{0}}^{z_{-}}\left(\zeta^{\prime}(t)\right)^{2} d t .
$$

Expanding the integrand in the $w$-plane and using the Cauchy-Riemann equations judiciously, we ultimately derive

$$
\bar{z}_{+}=\bar{z}_{0}+\frac{-h^{2} \psi_{v}^{2}+i h^{3} \psi_{v} \psi_{u v}}{z_{-}-z_{0}},
$$

correct to order $O\left(h^{3}\right)$. (See [9] for details.) We propose to use the approximate equation (42) as our reflection rule along $B C$.

Since this formula involves the intermediate point $z_{0}$ as well as $z_{+}$and $z_{-}$, and since it is only an approximation, the analysis of Part 1 does not apply and we must reconsider the implications of Eq. (42) as the mesh size $h$ goes to zero.

If we subtract $\bar{z}_{0}$, multiply by $\left(z_{-}-z_{0}\right)$, divide by $h^{2}$, and take the real part in (42), we get

$$
\operatorname{Re} \frac{\bar{z}_{+}-\bar{z}_{0}}{h} \frac{z_{-}-z_{0}}{h}=-\psi_{v}^{2} .
$$

Assuming convergence as $h \rightarrow 0$, we can identify the derivative $z_{v}$ in (43) and we have shown that the free boundary condition (28) is a consequence of (42) (remember $m=0$ ). 
There is another condition imposed by this rule, corresponding to the imaginary part of this equation. With a little algebraic manipulation we can derive

$$
\operatorname{Im} \frac{\bar{z}_{+}-2 \bar{z}_{0}+\bar{z}_{-}}{h^{2}} \frac{z_{-}-z_{0}}{h}=\psi_{v} \psi_{u v}
$$

which in the limit becomes

$$
-\operatorname{Im} \overline{z_{v v}} z_{v}=\psi_{v} \psi_{u v}
$$

This is just the $u$-derivative of Eq. (28), which holds all along $B C$; the substitution $z_{u}=-i z_{v}$ follows from the Cauchy-Riemann equations. Incidentally, notice that if we had not kept the $h^{3} \psi_{v} \psi_{u v}$ term in (42), we would be imposing the condition $\operatorname{Im} \overline{z_{v v}} z_{v}=0$ along $\Sigma$, which would be wrong. This is the reason for carrying the expansion of Eq. (41) so far.

Although we have shown that the consequences of Eq. (42) are consistent with our solution, they do not appear to be as strong as the ones generated by application of the exact reflection laws; namely, $z$ maps boundary to boundary, and Eq. (3). In fact, numerical experiments on a simple plasma-containment problem indicated that the application of the inexact rules does not determine a unique solution to the difference equations, as does the exact formulation with four-point normalization. Also, some of the solutions calculated with the inexact rule allowed the derivatives $z_{u}$ and $z_{v}$ to become nonorthogonal, violating (3).

Further experimentation led to the suspicion that the latter fault was the crucial defect of the procedure. The difficulty is resolved by modifying the reflection rule in such a way as to enforce the orthogonality of these derivatives. One can accomplish this by a adding an appropriate function of the derivatives. The new term should have the following properties:

(a) It should cause $z_{+}$to be shifted in a direction so as to make $z_{u}$ and $z_{v}$ more nearly perpendicular, and the amount of the shift should be greater when the nonorthogonality, as measured by $x_{u} x_{v}+y_{u} y_{v}$, is greater.

(b) It should be zero when $x_{u} x_{v}+y_{u} y_{v}$ is zero, so that under this condition the term has no effect and the two consequences of the reflection rule will hold.

(c) It must not cause instability, or else the iterates will still diverge.

The first two considerations immediately suggest that the term be proportional to $x_{u} x_{v}+y_{u} y_{v}$, which, of course, depends on the cosine of the angle between $z_{u}$ and $z_{v}$.

A study of the different geometrical possibilities involved ultimately shows that $\bar{z}_{+}$, as defined by (42), should be shifted an additional amount proportional to, and in the direction of, $i\left(x_{u} x_{v}+y_{u} y_{v}\right) /\left(z_{-}-z_{0}\right)$ (cf. [9]). We must incorporate this orthogonalitycorrecting term into (42), acknowledging the following consideration: the shift in $z_{+}$ should be of higher order than $z_{+}-z_{0}$ in (42). Otherwise, it may become the dominant term and obscure the free boundary constraint (28); furthermore, since tangential derivatives are involved, this term may tend to produce instability. Thus, we are led to the conviction that the correction, as formulated, should be multiplied by $h^{3}$; the modified reflection rule now becomes 


$$
\bar{z}_{+}=\bar{z}_{0}+\frac{-h^{2} \psi_{v}^{2}+i h^{3} \psi_{v} \psi_{u v}}{z_{-}-z_{0}}+\lambda h^{3} \frac{i\left(x_{u} x_{v}+y_{u} y_{v}\right)}{z_{-}-z_{0}},
$$

where $\lambda$ is a constant to be chosen experimentally.

The value of $\lambda$ which gives good results for all the problems considered and all mesh sizes seems to be about 50 . However, experimentation has revealed that this type of reflection law, coupled with an overrelaxation technique to solve the equations, is not very sensitive to the particular value of $\lambda$ used. Evidently, the correction successfully achieves our goal, i.e., the enforcement of Eq. (3), so that the solution is independent of $\lambda$.

For the three-dimensional case, we could proceed as before and derive the reflection rule from Garabedian's integral equation, but we prefer to simply try to modify the two-dimensional rule in a suitable manner. We can get the proper factors of $y$ into the resulting conditions, as $h$ goes to zero, by using the formula

$$
\bar{z}_{+}=\bar{z}_{0}+\frac{-h^{2} \frac{\psi_{v}^{2}}{y^{2}}+i \frac{h^{3}}{2} \frac{\partial}{\partial u}\left(\frac{\psi_{v}}{y}\right)^{2}}{z_{-}-z_{0}}+\frac{\lambda h^{3} i\left(x_{u} x_{v}+y_{u} y_{v}\right)}{z_{-}-z_{0}} .
$$

Reasoning as before, we see that Eq. (28), its $u$-derivative, and Eq. (3) all follow from (47).

We emphasize here that we have just arrived at a reflection rule by a heuristic reasoning process quite different from the procedure outlined in Part 1, or from the integral equation of Garabedian. We do not know the location of $\Sigma$ (i.e., Eq. (8)), and we have not invoked the analyticity of the curve, yet we are able to use reflection to express the boundary conditions. This demonstrates the possibility of applying the method to a very general class of problems.

We conclude that using the above approximate reflection rules (46) and (47) should insure the free boundary condition (28) and orthogonality (3) along $B C$. Clearly, using approximate rules requires more care than the exact formulation (8). First, we can only be sure of (3) if our solution is insensitive to the value of $\lambda$; this must be tested. Second, we have no direct proof that $z(w)$ will map boundary to boundary; our argument for this is based on the observation that the solution to the difference equations seems to be unique, as indicated by the fact that the overrelaxed iterations of the solution do converge, while we would expect them to oscillate if there were more than one solution. Since our equations are certainly consistent, our solution must be the right one. Third, we still have the problem, inherent in any reflection scheme using four-point normalization, that $H=x_{v}^{2}+y_{v}^{2}-x_{u}^{2}-y_{u}^{2}$ need not be zero; this will be taken care of in section $\mathrm{G}$.

F. The Separation Point. Some additional thought must be given to the separation point $B$, in order to preserve the second-order accuracy criteria employed in Eqs. (46) and (47).

First of all, the expansion of $\psi(z)$ near $i Y_{0}$ occurs in powers of $\left(z-i Y_{0}\right)^{1 / 2}$ (cf. [4] ). This results in lower accuracy for the Laplace difference equation (5) in the $z$-plane. However, we have already corrected this by our choice of geometry; $z(w)$ maps 
the $90^{\circ}$ corner at $B$ to the $180^{\circ}$ separation point $i Y_{0}$, so $z$ expands in power of $(w-i)^{2}$, and the accuracy of the finite-difference equation for $\psi$ is restored to $O\left(h^{2}\right)$ in the $w$-plane.

A more troublesome inaccuracy arises from the reflection rule (46) or (47). Since $\psi \equiv 1$ on $A B$ and $z(w)$ has a branch point at $B$, both $\psi_{v}$ and $z_{-}-z_{0}$ are $O(h)$ and one of the terms becomes $0 / 0$ at $B$. The rule (30) for reflecting through the wall is exact; we must repair (46) and (47). The geometry near $B$ is shown in Fig. 4, where image points are identified by their subscripts. As $h \rightarrow 0$, the pairs of points $z_{1}$ and $z_{2}, z_{4}$ and $z_{5}$, and $z_{6}$ and $z_{7}$ coalesce because of the branch point.
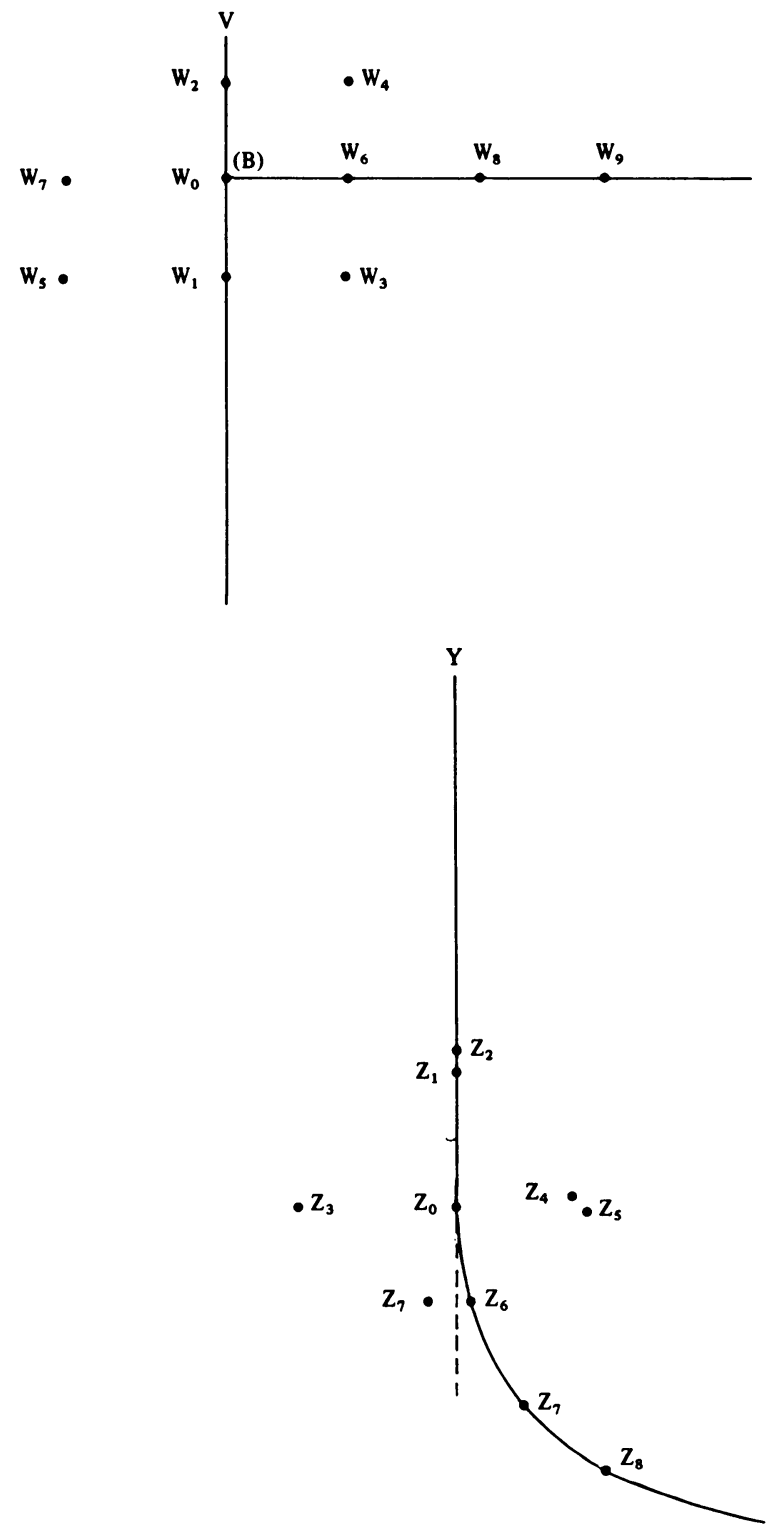

FIGURE 4

For the two-dimensional case, we return to the integral formula (41); using the notation of Fig. 4, we write it as 


$$
\bar{z}_{2}-\bar{z}_{0}=\int_{z_{0}}^{z_{1}}\left(\zeta^{\prime}\right)^{2} d z
$$

As we indicated above, $\zeta$ has an expansion in powers of $\left(z-z_{0}\right)^{1 / 2}$. More explicitly,

$$
\zeta=\zeta_{0}+A_{1}\left(z-z_{0}\right)^{1 / 2}+A_{2}\left(z-z_{0}\right)+A_{3}\left(z-z_{0}\right)^{3 / 2}+\cdots,
$$

where $A_{1}$ is zero (cf. [5]). This gives $\zeta^{\prime}(z)=\zeta^{\prime}\left(z_{0}\right)+O\left(\left|z-z_{0}\right|^{1 / 2}\right)$ so we can write

$$
\bar{z}_{2}-\bar{z}_{0}=\zeta_{0}^{\prime 2}\left(z_{1}-z_{0}\right)+O\left(\left|z_{1}-z_{0}\right|^{3 / 2}\right) \text {. }
$$

Since $z_{0}$ is the separation point, $z_{0}^{\prime}=0$. Thus, $z_{1}-z_{0}$ is $O\left(h^{2}\right)$ and the error here is $O\left(h^{3}\right)$, while the left-hand side is $O\left(h^{2}\right)$. Also, we have

$$
\zeta_{0}^{\prime}=d(\phi+i \psi) / d z=\phi_{x}+i \psi_{x}
$$

but analyticity requires $\phi_{x}=\psi_{y}=0$ on $A B$, so $\zeta_{0}^{\prime}=i \psi_{x}$ and the integral representation yields

$$
\bar{z}_{2}-\bar{z}_{0}=-\left(z_{1}-z_{0}\right) \psi_{x}^{2}+O\left(h^{3}\right)
$$

In order to use this as a reflection rule, we must find a sufficiently accurate approximation for $\psi_{x}$ at $z_{0}$. We expand $\psi$ around $z_{0}$ to get $\psi\left(x_{3}, y_{3}\right)=\psi_{3}$; namely,

$$
\psi_{3}=\psi_{0}+\psi_{x}\left(x_{3}-x_{0}\right)+\psi_{y}\left(y_{3}-y_{0}\right)+O\left(\left|z_{3}-z_{0}\right|^{3 / 2}\right)
$$

(see Fig. 4). Using the above estimates, we derive

$$
\psi_{x}=\left(\psi_{3}-\psi_{0}\right) /\left(x_{3}-x_{0}\right)+O(h) .
$$

Therefore, we take for our reflection law at the corner $B$,

$$
\bar{z}_{2}=\bar{z}_{0}+\left(z_{0}-z_{1}\right)\left(\frac{\psi_{3}-\psi_{0}}{x_{3}-x_{0}}\right)^{2},
$$

accurate to order $O\left(h^{3}\right)$.

We must now examine the consequences of this rule. As $h \rightarrow 0$, it becomes

$$
\overline{z_{v}}=z_{v} \psi_{x}^{2}
$$

From this we conclude that either

$$
\operatorname{Im} z_{v}=0 \quad \text { and } \quad \psi_{x}^{2}=1
$$

or

$$
z_{v}=0
$$

But $x \equiv 0$ on $A B$, so $x_{v}=0$, and either conclusion leads to (58).

With this in mind we look at the higher order limits and get

$$
\bar{z}_{v v}=-z_{v v} \psi_{x}^{2}
$$

Again we are led to alternatives; if $z_{v v}$ is zero, we take still higher order terms until we 
get a nonzero derivative $z_{v v} \ldots v$, at which point we finally conclude

$$
\psi_{x}^{2}=1
$$

Thus, we see that the consequences of (55) are (58) and (59). Clearly, (58) is consistent; and since $\psi_{x}$ is the normal derivative of $\psi$ in the $z$-plane at the separation point, the second equation agrees with (23).

For the axially symmetric case we modify (55) to become

$$
\bar{z}_{2}=\bar{z}_{0}+\left(z_{0}-z_{1}\right)\left(\frac{\psi_{3}-\psi_{0}}{x_{3}-x_{0}}\right)^{2} \frac{1}{y^{2}} .
$$

Analogous calculations show that this is consistent with (58) and (23), also.

G. The Line of Truncation. The values of $x, y$, and $\psi$ on the line $C D$ will approach their asymptotic limits as the length of the rectangle, $A D$, is increased. So as an approximation we set

$$
y=v, \quad x=x_{1}=\text { constant, and } \psi=v^{m+1} /(m+1)
$$

on $C D$. (Later we shall extrapolate our results for the length $A D$ going to infinity.) The choice of the number $x_{1}$ is determined by the following considerations.

Recall that no provision has yet been made for driving $H=x_{v}^{2}+y_{v}^{2}-x_{u}^{2}-$ $y_{u}^{2}$ to zero. Since $x_{1}$ is the only undetermined parameter in the problem, we use it to enforce this condition. Experimentation on several models has indicated that the solution to the difference equations is unique for any fixed $x_{1}$, so $H$ can be regarded as a function of $x_{1}$. We, therefore, use a proportional-gain procedure. While we are iteratively solving the equations, we compute $H$, and then shift $x_{1}$ by an amount

$$
\delta x_{1}=\mu H \text {. }
$$

Heuristic arguments (cf. [9]) indicate that the constant $\mu$ should be positive, and approximately equal to 2 .

To actually implement this procedure we use the following scheme, which is designed to enhance the stability. The value of $H$ is computed by centered differences at all points in the rectangle $A B C D$ to the right of $u=1$ (thus excluding the pole) and the average is formed. Then $x_{1}$ is shifted according to (62). In addition, the $x$-coordinates of all the mesh points involved in this average are shifted by a proportional amount. This operation is not performed after every iteration of the equations, but rather, we wait an appropriate number of iterations after shifting $x_{1}$ for the effect to settle.

Experimentation shows that this scheme does drive $H$ to zero. The optimal number of iterations between shifts seems to be the same as the number of mesh points along the $x$-axis, and the best value of $\mu$ is

$$
\mu=2.8 \text {. }
$$

H. The Residue at the Origin. The residue $R$ of $z(w)$ at $w=0$, appearing in 
Eq. (35), is determined by the condition of analyticity; we have already specified the images of three points of the rectangle, namely, $A, B$, and $C$ (recall $Y_{\infty}=1$ ), so we have no freedom left to choose $R$. The velocity-averaging method described in [4] for overcoming the difficulty seems to this author to be somewhat obscure, so we invented an altogether different, if not better motivated, technique.

Referring to Fig. 3, the reader may expect that if the residue were too big, the flow region would be to some extent "sucked out" to infinity in the second quadrant, drawing the free surface (which is the only part of the boundary not "rigidly" fixed) down and in; while the opposite would happen if $R$ were too small. The result would be a nonzero angle of separation at $z=i Y_{0}$. Numerical experiments have completely confirmed this heuristic guess; the equations were solved for different fixed values of $R$, and it was found that the angle of detachment was a monotonic function of the parameter $R$.

This suggests another proportional-gain computation like that in section $\mathrm{G}$; at the same time we are correcting the value of $x_{1}$ by Eq. (62), we adjust $R$ by an amount proportional to the angle formed at detachment. The most successful scheme appears to be the following.

Let us examine the expansion of $z(w)$ along $B C$, near the point $B$ (where $w=i$ ).

$$
\begin{aligned}
& x(u+i)=x_{B}+\left.\frac{\partial x}{\partial u}\right|_{B} u+\left.\frac{\partial^{2} x}{\partial u^{2}}\right|_{B} \frac{u^{2}}{2}+\left.\frac{\partial^{3} x}{\partial u^{3}}\right|_{B} \frac{u^{3}}{3 !}+O\left(u^{4}\right), \\
& y(u+i)=Y_{0}+\left.\frac{\partial y}{\partial u}\right|_{B} u+\left.\frac{\partial^{2} y}{\partial u^{2}}\right|_{B} \frac{u^{2}}{2}+O\left(u^{3}\right) .
\end{aligned}
$$

For the exact solution, $x_{B}=0$ and both first derivatives vanish (since $B$ is a branch point). Furthermore, $x$ is identically zero on $A B$ so $x_{v v}=0$, and harmonicity implies

$$
\left.\frac{\partial^{2} x}{\partial u^{2}}\right|_{B}=0
$$

also. Thus,

$$
x=O\left(u^{3}\right) \text { and } y=Y_{0}+O\left(u^{2}\right)
$$

which displays the smooth detachment property explicitly.

Now in our approximate difference scheme, $x_{B}$ is made zero by the reflection law on $A B . x_{u u}$ is driven to zero by equating it, through Eq. (5), with $x_{v v}$, which is forced to zero by the same reflection law. There is no such explicit mechanism for the term $x_{u}$. We shall, therefore, use the computed value of $x_{u}$ as a measure of the angle of detachment. This value will be calculated from the approximate formula (see Fig. 4)

$$
\left.x_{\mu}\right|_{B}=\left(3 x_{6}-\frac{3}{2} x_{8}+\frac{1}{3} x_{9}\right) / h+O\left(h^{3}\right),
$$

which is exact for cubic polynomials, and thus is most appropriate because of (66). 
Therefore, every time we change $x_{1}$ according to (62), we also adjust $R$ by the amount

$$
\delta R=\left.\eta x_{u}\right|_{B}
$$

The constant $\eta$ is chosen experimentally to achieve optimal convergence for the (overrelaxed) iterates of the equations, but the final solution must be insensitive to changes in $\eta$ if we are to conclude $x_{u}=0$. The value $\eta=30$ seems to work best in this model.

I. Initialization and Iteration. The initial values for $x, y$, and $\psi$ are chosen to be the exact solutions for the two-dimensional case. These can be found by the hodograph technique, and are given by

$$
\begin{aligned}
& \psi=\operatorname{Im} \frac{2}{\Pi} \zeta \\
& z=2 i-\frac{2}{\Pi}\left\{\zeta+e^{-\zeta}+\left(1+e^{-2 \zeta}\right)^{1 / 2}-\log \left(1-\left(1+e^{-2 \zeta}\right)^{1 / 2}\right)\right\}
\end{aligned}
$$

where

$$
\zeta=\sin (\Pi w / 2)
$$

Care must be exercised in the choice of quadrant for the square root.

With these starting values, the system of equations can be solved iteratively using the overrelaxation technique. The results of computer runs are given in Part 3. We summarize by presenting an outline of the iterative procedure.

A basic iteration begins with stored values of $x, y$ and $\psi$. First the reflected values of $x$ and $y$ are computed according to (30) on $A B,(31)$ on $A D,(46)$ or (47) on $B C$, and (55) or (60) at the corner $B$. The values on $C D$ are retained as per Eq. (61), and the values of $\psi$ on the other three sides are fixed in accordance with the normalizations (21) and (22).

Next we update the interior values of $x$ and $y$ by successive overrelaxation. If we denote the right-hand side of Eq. (5) by $x^{\prime}$, the update proceeds according to the rule

$$
x_{\mathrm{new}}=x_{\mathrm{old}}+\omega\left(x^{\prime}-x_{\mathrm{old}}\right)
$$

where the relaxation factor $\omega$ is computed by $\omega=2 /(1+\alpha h)$ (cf. [3]). Experimentation resulted in an optimal choice (in terms of speed of convergence) of $\alpha=12$. The values of $y$ are updated similarly. The updates proceed successively, i.e. new values are used in (5) as they are computed, in a straightforward up-and-down left-to-right order. The largest residual $\left|x_{\text {new }}-x_{\text {old }}\right|$ that occurs is noted and used in determining convergence.

Because the boundary conditions for $\psi$ are so much less complicated, its iterates converge much faster than those of $x$ and $y$. Consequently, $\psi$ is only updated every ten iterations. The same procedure is followed, except that Eq. (29) is used for $\psi^{\prime}$ in the axisymmetric case, and the optimal relaxation parameter appears to be $\alpha=4$.

Of course, the transformations (32)-(35) must be observed near the corner $A$. 
The computation indicated in Eq. (61) is performed once every $N$ iterations, where $N$ is the number of mesh points along $A D$. Similarly, the residue correction (68) is performed once every $N$ iterations.

Finally, once the convergence criterion is established (by testing the maximal residual, last shift in $x_{1}$, and last residue correction), the parameters $\lambda, \mu$ and $\eta$ are doubled and the iterations begin again, to test the insensitivity of the answers with respect to these "ad hoc" numbers.

If more accuracy is desired, the mesh size is then halved, new values for $x, y$ and $\psi$ are interpolated, and we start over.

A crude comparison of the computational effort per iteration between the reflection scheme and the steepest-descent method, based on the number of Fortran instructions, indicates that they are about the same. Also, the amount of relaxation used is roughly equal.

Part 3. Results. The procedure of Part 2 was programmed and run on New York University's CDC 6600 computer for many test cases. Here we describe the results with regard to accuracy and convergence.

Throughout Part 3 we shall compare our results with those of Bloch [4], because a measure of the success of the reflection procedure is the degree to which it improves on the efficiency of the method of steepest descent. The superiority of the reflection technique is clearly demonstrated in section A by computer runs requiring about onetenth the number of iterations needed for analogous cases employing steepest descent.

A. Rate of Convergence of the Iterations. For the two-dimensional vena contracta model we choose a $w$-rectangle of dimension $41 / 3$ by 1 , with mesh size $h=$ 1/48. This involves 10,708 mesh points. The program was iterated until all residuals were less than $10^{-6}$. The number of cycles required was 588. For comparison, Bloch quotes a similar run with a 6 by 1 rectangle $(14,161$ mesh points); it required 6,260 iterations to achieve the same accuracy.

For the axially symmetric case the same rectangle was employed but the initialization was slightly altered. We used the exact two-dimensional data with $h=1 / 6$. After residuals were reduced to $10^{-5}$, the mesh was halved and the data interpolated for the new points. This was repeated, but for $h=1 / 48$ the iterations proceeded until the residuals were less than $10^{-6}$. The number of iterations required was

$\begin{array}{cc}\text { Number of Iterations } & \text { Mesh Size } \\ 257 & 1 / 6 \\ 162 & 1 / 12 \\ 851 & 1 / 24 \\ 946 & 1 / 48\end{array}$

We mention that as $h$ was decreased, the amount of overrelaxation used was increased extremely rapidly.

For comparison, Bloch quotes 13,368 iterations required to reduce residuals to 
$10^{-6}$ in this case with 10,633 mesh points.

We conclude that the reflection scheme requires about one-tenth as many iterations as steepest descent for the same degree of convergence.

B. Accuracy. All the approximate equations employed in this program are accurate to second order (that is, the order of the truncated terms exceeds the order of the rest of the equation by 2), so we may expect that if centered differences are used the solutions ought to converge like $O\left(h^{2}\right)$ as $h \rightarrow 0$. This was confirmed by the experiments; the values computed for the contraction coefficients (25) using the $41 / 3$ by 1 rectangle described above, for different mesh sizes, are excellently approximated by

$$
\begin{array}{ll}
C_{c}(h)=.61005-.21 h^{2} & (m=0), \\
C_{c}(h)=.59133+.70 h^{2} & (m=1) .
\end{array}
$$

Of course, these extrapolated values must still be corrected for the finite length of the rectangle (see below).

C. Dependence on Experimental Parameters; The Contraction Coefficients. There are four parameters in our vena contracta model which are chosen experimentally to achieve certain goals. They are:

$$
\begin{aligned}
& \lambda \text { (Eqs. (46) and (47)), } \\
& \mu \text { (Eq. (62)), } \\
& \eta \text { (Eq. (68)), and } \\
& U_{\text {max }} \text {, the length of the rectangle } A B C D .
\end{aligned}
$$

The values for the first three are chosen to speed convergence, but because they come from proportional-gain errors, the final solutions must be insensitive to them. We tested this by doubling their values after all residuals were less than $10^{-6}$; the resulting perturbation needed only at most $10 \%$ additional iterations to reproduce this convergence criteria, and the contraction coefficients were changed by no more than one unit in the fourth decimal place. Evidently their utilizations were successful. Bloch [4] has argued that the effect of truncating the rectangle is to change the contraction coefficient according to

$$
C_{c}\left(U_{\max }\right)=C_{c}-A e^{-\lambda U_{\max }}
$$

where $A$ and $\lambda$ are constants. Our observations fit this formula, with the values

$$
\begin{array}{ccc} 
& m=0 & m=1 \\
A & .7 & 1.2 \\
\lambda & 1.5 & 2.2
\end{array}
$$

Extrapolating our data for all these corrections, we are led to the following estimates of the contraction coefficients:

$$
C_{c}=.61106, \quad m=0 \text { and } C_{c}=.59142, \quad m=1,
$$

where at least the fourth decimal is significant. 
For comparison, Bloch [4] quotes

$$
C_{c}(0)=.61100 \pm .00002, \quad C_{c}(1)=.59135 \pm .00004,
$$

and our computations confirm these values. The exact value for $m=0$, is, from (68),

$$
C_{c}(0)=\pi /(\pi+2)=.611015 \ldots
$$

Department of Mathematics

University of South Florida

Tampa, Florida 33620

1. H. LEWY, "On the reflection laws of second order differential equations in two independent variables," Bull. Amer. Math. Soc., v. 65, 1959, pp. 37-58. MR 21 \#210.

2. V. FILLIPENKO, "On the reflection of harmonic functions and of solutions of the wave equations," Pacific J. Math., v. 14, 1964, pp. 883-893.

3. P. R. GARABEDIAN, Partial Differential Equations, Wiley, New York, 1964. MR 28 \#5247.

4. E. BLOCH, A Finite Difference Method for the Solution of Free Boundary Problems, AEC Computing and Applied Mathematics Center, Courant Inst. Math. Sci., New York Univ., NYO-1480-116, 1969.

5. D. GILBARG, Jets and Cavities, Handbuch der Physik, vol. 9, part 3, Springer-Verlag, Berlin, 1960. MR 22 \#10416.

6. Z. NEHARI, Conformal Mapping, McGraw-Hill, New York, 1952. MR 13, 640.

7. D. M. YOUNG, "Iterative methods for solving partial differential equations of elliptic ty pe," Trans. Amer. Math. Soc.,v. 76, 1954, pp. 92-111. MR 15, 562.

8. J. H. GIESE, "On the truncation error in a numerical solution of the Neumann problem for a rectangle," J. Math. Phys.,v. 37, 1958, pp. 169-177. MR 20 \#2845.

9. A. D. SNIDER, Numerical Solution of Nonlinear Boundary Value Problems Using Reflection, AEC Computing and Applied Mathematics Center, Courant Inst. Math. Sci., New York Univ., NYO-1480-167, 1971.

10. P. R. GARABEDIAN, "Calculation of axially symmetric cavities and jets," Pacific J. Math., v. 6, 1956, pp. 611-684. MR 19, 348. 